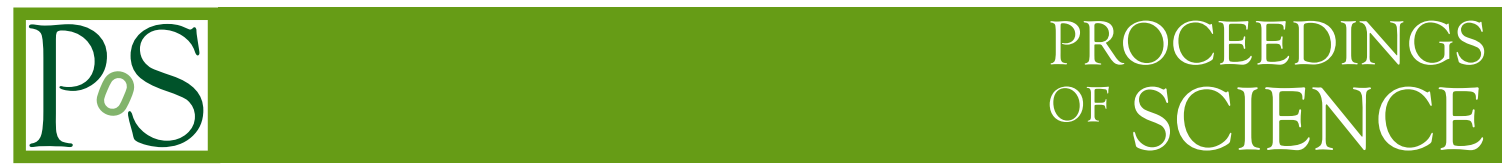

\title{
Measurement of $\gamma$ from $B$ meson decay to $D^{(*)} K^{(*)}$
}

\author{
Frédéric Machefert ${ }^{*} \dagger$ \\ LAL, Université Paris-Sud, CNRS/IN2P3, Orsay, France. \\ E-mail: frederic.machefertein2p3.fr
}

\begin{abstract}
The LHCb experiment has measured the angle $\gamma$ of the unitarity triangle using $B \rightarrow D^{(*)} K^{(*)}$ decays and with an integrated luminosity data sample of up to $4 \mathrm{fb}^{-1}$ at energies in the centerof-mass of 7, 8 and $13 \mathrm{TeV}$. Many modes have been used, but the most recent results have been obtained with the GLW and ADS two-body decays and their extensions, the quasi-GLW and quasi-ADS modes. They are described in this paper and the results obtained by the $\mathrm{LHCb}$ collaboration are given. The $\gamma$ angle measurement of LHCb is the combination of many modes among which the charged $B$ decays contributed, and the most precise measurement from a single experiment.
\end{abstract}

9th International Workshop on the CKM Unitarity Triangle

28 November - 3 December 2016

Tata Institute for Fundamental Research (TIFR), Mumbai, India

\footnotetext{
${ }^{*}$ Speaker.

${ }^{\dagger}$ On behalf of the LHCb collaboration.
} 


\section{Introduction}

The angle $\gamma$ of the unitarity triangle of the Cabibbo Kobayashi Maskawa (CKM) matrix,

$$
\gamma \equiv \arg \left(-\frac{V_{u d} V_{u b}^{\star}}{V_{c d} V_{c b}^{\star}}\right) \approx \arg \left(-\frac{V_{u b}^{\star}}{V_{c b}^{\star}}\right)
$$

can be measured when $b \rightarrow u$ and $b \rightarrow c$ decay types interfere and potentially exhibit a sizable $\mathrm{CP}$ violation. The measurement can be done without penguin pollution (no $V_{t x} \mathrm{CKM}$ terms are involved), at the tree level and with small theoretical uncertainties [1]. The $B \rightarrow D^{(*)} K^{(*)}$ decays satisfy those criteria and some give a large $\mathrm{CP}$ violation.

The combination of the direct measurements by Babar, Belle and LHCb gives $\gamma=\left(73.2_{-7.0}^{+6.3}\right)^{\circ}$ and the present indirect determination of $\gamma$ is $\left(66.85_{-3.44}^{+0.94}\right)^{\circ}$. Hence, the improvement of the precision of the direct measurement [2] is crucial and the LHCb collaboration aims at reaching the degree-level precision in the future.

\subsection{The observables}

The method consists in having an interference between two $B$ meson decay paths of type $b \rightarrow u \bar{c} s$ and $b \rightarrow c \bar{u} s$, leading to the same final state of the $D^{0}$ and $\bar{D}^{0}$ mesons (see Figure 1). The
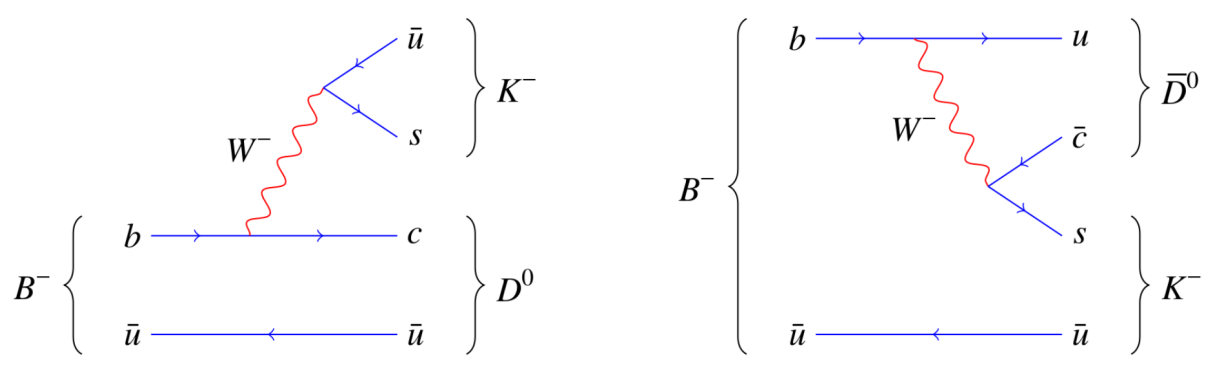

Figure 1: Feynman diagrams of the two interfering processes $B^{-} \rightarrow D K^{-}$, where the $D$ meson decays into a unique final state.

$\mathrm{CP}$ violation observables that are used to measure $\gamma$ in the modes described in this paper, are the charge asymmetries $A$ and partial width ratios $R$ of the decays,

$$
A=\frac{\Gamma\left(B^{-} \rightarrow f_{D} K^{-}\right)-\Gamma\left(B^{+} \rightarrow \bar{f}_{D} K^{+}\right)}{\Gamma\left(B^{-} \rightarrow f_{D} K^{-}\right)+\Gamma\left(B^{+} \rightarrow \bar{f}_{D} K^{+}\right)}, R=\frac{\Gamma\left(B^{-} \rightarrow \bar{f}_{D} K^{-}\right)+\Gamma\left(B^{+} \rightarrow f_{D} K^{+}\right)}{\Gamma\left(B^{-} \rightarrow f_{D} K^{-}\right)+\Gamma\left(B^{+} \rightarrow \bar{f}_{D} K^{+}\right)}
$$

The observables depend essentially on the ratio of the two interfering amplitudes that can be expressed from the amplitude ratio $r_{B}$, the strong phase difference $\delta_{B}$ and $\gamma$,

$$
\frac{A\left(B^{-} \rightarrow \bar{D}^{0} K^{-}\right)}{A\left(B^{-} \rightarrow D^{0} K^{-}\right)}=r_{B} e^{i\left(\delta_{B}-\gamma\right)}
$$

The results presented in this paper have been published in [3,9] 


\subsection{GLW and ADS methods}

Different decay modes can be used to be sensitive to the amplitude interferences. The GLW method (for Gronau, London and Wyler [5, 14]), is based on the D meson decay in a CP-eigenstate (see 2, left). The D meson decay amplitude ratio and phase are simplified, however the interference is potentially small due to the large mis-balance of the amplitudes of the decay. The ADS method (for Atwood, Dunietz and Soni [7]) is based on favoured and suppressed $B$ and $D$ meson decays in both interfering amplitudes leading to a more balanced process, larger interferences and asymmetries (see 2, right). However, the amplitude ratio and phase difference of the $D$ meson decay is an input to this method. The partial width in the two methods is easily expressed in term of the
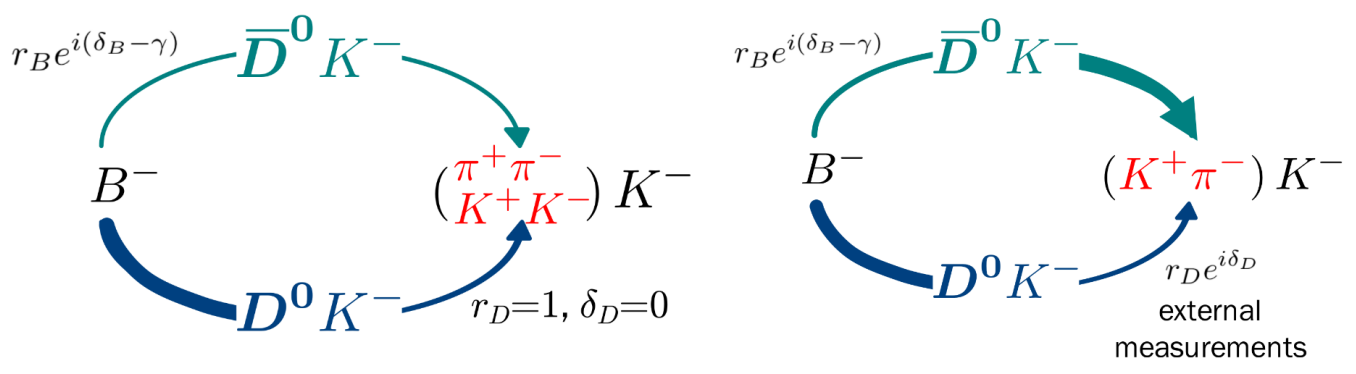

Figure 2: Schematics of the GLW (left) and ADS (right) decay modes altogether with the corresponding main parameters.

amplitude ratio(s) of the $B$ meson (and the $D$ meson for the ADS mode), the phase difference(s) and $\gamma$,

$$
\begin{aligned}
& \text { GLW : } \Gamma\left(B^{ \pm} \rightarrow f_{D} K^{ \pm}\right) \propto 1+r_{B}+2 r_{B} \cos \left(\delta_{b} \pm \gamma\right) \\
& \text { ADS : } \Gamma\left(B^{ \pm} \rightarrow f_{D} K^{ \pm}\right) \propto\left(r_{D}^{f}\right)^{2} r_{B}^{2}+2 r_{B} r_{D}^{f} \cos \left(\delta_{b}+\delta_{D}^{f} \pm \gamma\right) .
\end{aligned}
$$

\subsection{Quasi-GLW and quasi-ADS methods}

The two-body decay of the GLW and ADS methods has been extended to four-body decays where the $D$ meson produces either a $\pi^{+} \pi^{-} \pi^{+} \pi^{-}$or a $K^{+} \pi^{-} \pi^{+} \pi^{-}$final state. The former, or quasi-GLW mode, does not produce a strict CP-eigentate and is polluted by a fractional CP-even content which has been measured to be $F_{+}=0.737 \pm 0.028[8,9]$. Similarly, the latter, or quasiADS mode, is diluted by the $\kappa_{D}^{3 \pi}$ coherence factor of the $D$ decay which has been measured to be $0.43_{-0.13}^{+0.17}[10,11]$. The partial width are modified as follows,

$$
\begin{aligned}
& \text { GLW : } \Gamma\left(B^{ \pm} \rightarrow f_{D} K^{ \pm}\right) \propto 1+r_{B}+\left(2 F_{+}-1\right) 2 r_{B} \cos \left(\delta_{b} \pm \gamma\right) \\
& \text { ADS : } \Gamma\left(B^{ \pm} \rightarrow f_{D} K^{ \pm}\right) \propto\left(r_{D}^{f}\right)^{2} r_{B}^{2}+2 r_{B} r_{D}^{f} \kappa_{D}^{f} \cos \left(\delta_{b}+\delta_{D}^{f} \pm \gamma\right) .
\end{aligned}
$$

\subsection{Other methods}

There exit other methods to measure $\gamma$ which have been used by LHCb, the final measurement combining the different determinations. The GLS method exploits singly Cabibbo suppressed decays like $D \rightarrow K_{s}^{0} K \pi$ and the GGSZ one uses self conjugate three-body decays (e.g. $D \rightarrow K_{s}^{0} \pi \pi$ ) and is based on the fit of the corresponding Dalitz figure. There have been no recent measurements with those modes and the corresponding results can be obtained from [12, 13]. 


\section{Results}

\section{1 (Quasi-)GLW modes}

Figure 3 shows the charged $B$ meson invariant mass distribution reconstructed from protonproton collision data corresponding to an integrated luminosity of $3.0 \mathrm{fb}^{-1}$ at center-of-mass energies of 7 and $8 \mathrm{TeV}$. The total $B$ meson yields in the $D \rightarrow K K$ and $D \rightarrow \pi \pi$ modes are respectively of $3816 \pm 92$ and $1162 \pm 48$ candidates. The asymmetries both in the $K K$ and $\pi \pi$ modes are
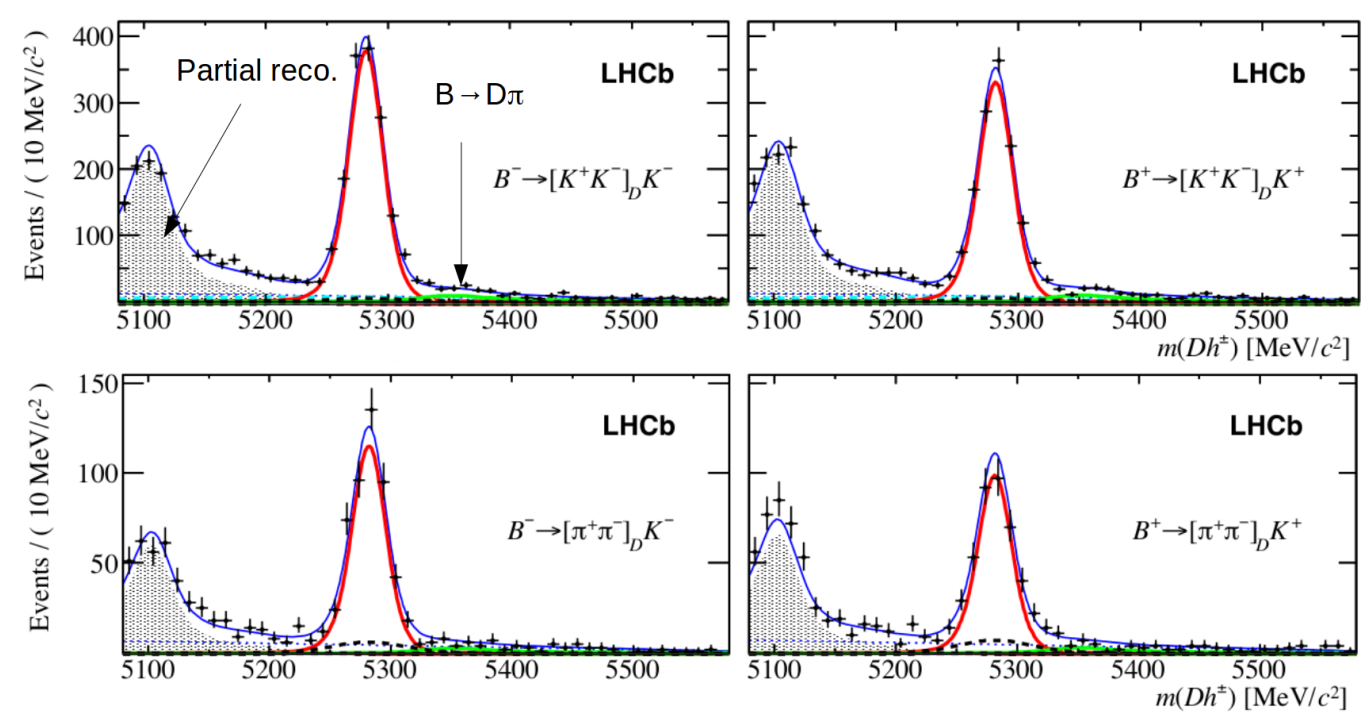

Figure 3: Invariant mass distribution of the $B$ meson reconstructed in the decays $B^{ \pm} \rightarrow\left[K^{+} K^{-}\right]_{D} K^{ \pm}$and $B^{ \pm} \rightarrow\left[\pi^{+} \pi^{-}\right]_{D} K^{ \pm}$. The partially reconstructed background is shown in hashed grey and the $B \rightarrow D K$ $(B \rightarrow D \pi)$ contribution in red (green). The small combinatorial contribution is indicated with the dotted line.

measured to be $A_{K}^{K K}=0.087 \pm 0.020 \pm 0.008$ and $A_{K}^{\pi \pi}=0.128 \pm 0.037 \pm 0.012$, respectively. In spite of the dilution due to the fractional CP-even content of the decay, whose amplitude reaches $\left(2 F_{+}-1\right) \sim 0.5$, the quasi-GLW mode gives an asymmetry of $A_{K}^{\pi \pi \pi \pi}=0.100 \pm 0.034 \pm 0.018$.

\section{2 (Quasi-)ADS modes}

The ADS modes are seen by LHCb, both in the two- and quasi-ADS four-body decays in the $3 \mathrm{fb}^{-1}$ data sample. The yield are of $29470 \pm 230$ candidates $B^{ \pm} \rightarrow\left[K^{ \pm} \pi^{\mp}\right]_{D} K^{ \pm}$and $553 \pm$ 34 candidates $B^{ \pm} \rightarrow\left[\pi^{ \pm} K^{\mp}\right]_{D} K^{ \pm}$for the two-body decays. This last mode is very rare and has been seen only by LHCb. The corresponding asymmetry in the $\pi K$ ADS decay is of $A_{A D S(K)}^{\pi K}=$ $-0.403 \pm 0.056 \pm 0.011$. This is the first observation of $\mathrm{CP}$ violation in a single $B \rightarrow D h$ mode, with a significance of $8 \sigma$ (see Figure 4). The yields for the quasi-ADS mode reach $11330 \pm 140$ candidates $B^{ \pm} \rightarrow\left[K^{ \pm} \pi^{\mp} \pi^{+} \pi^{-}\right]_{D} K^{ \pm}$and $159 \pm 17$ in the $B^{ \pm} \rightarrow\left[\pi^{ \pm} K^{\mp} \pi^{+} \pi^{-}\right]_{D} K^{ \pm}$channel. The asymmetry which is determined from those yields is $A_{A D S(K)}^{\pi K \pi \pi}=-0.313 \pm 0.102 \pm 0.038$. From the value of $\delta_{D}$ [11], a similar sign for the asymmetry is expected here as for the ADS two-body decay. 


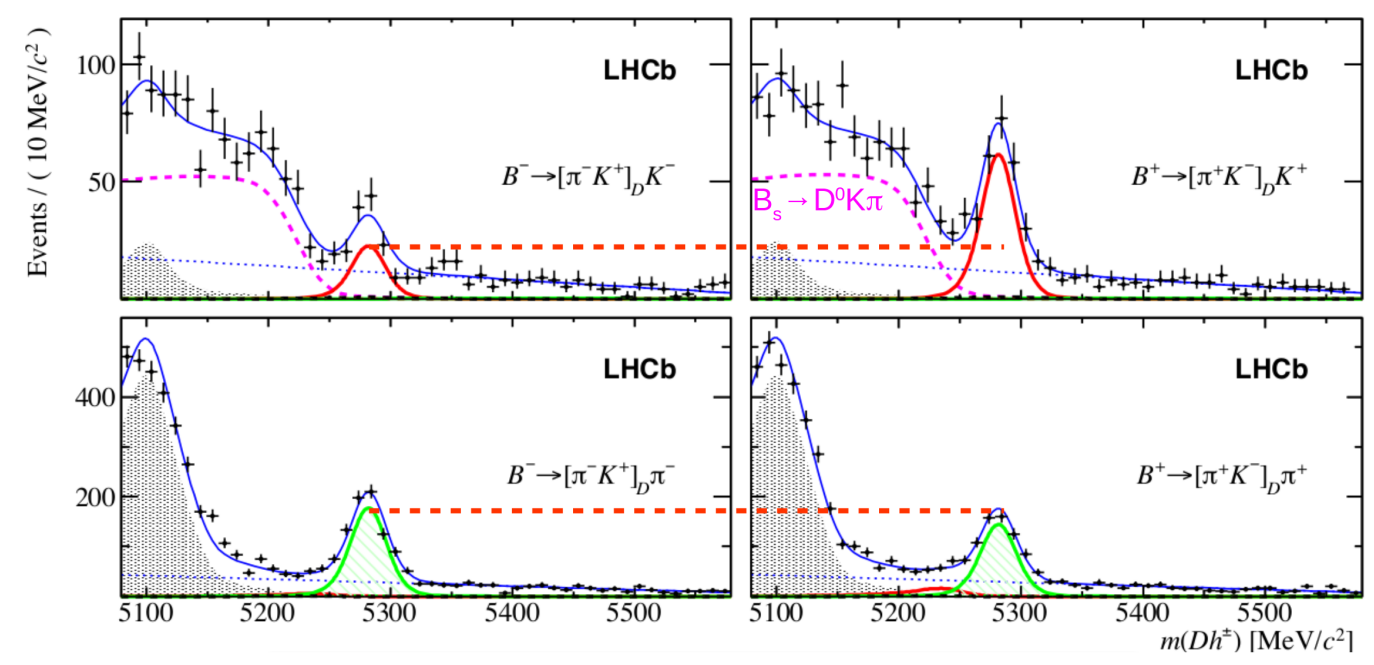

Figure 4: Invariant mass distribution of the reconstructed $B$ meson in the $B^{ \pm} \rightarrow\left[\pi^{ \pm} K^{\mp}\right]_{D} K^{ \pm}$channel (top). The red and green lines show the $D K$ and $D \pi$ contributions. The hashed grey area corresponds to the partially reconstructed background, and the dotted contribution to the combinatorial background. The large asymmetry observed is indicated by the red dotted line. The corresponding $B^{ \pm} \rightarrow\left[\pi^{ \pm} K^{\mp}\right]_{D} \pi^{ \pm}$is also shown (bottom).

\subsection{First results in the $B^{ \pm} \rightarrow D K^{\star \pm}$}

In this decay mode the same interference as in the previous sections can be used, in a new $B$ meson final state. Both the GLW and ADS techniques can be applied to measure asymmetries and partial width ratios. The LHCb experiment has published first results [16] based on an integrated luminosity of $4 \mathrm{fb}^{-1}$. However, the data sample is still limited, and is not large enough to exclude large regions of the parameter space. But the result is consistent with $\gamma \approx 70^{\circ}$.

\section{Conclusion}

The measurement of $\gamma$ with the LHCb experiment is the most precise measurement from a single experiment [15], with an uncertainty of the order of $7^{\circ}$. Figure 5 shows the determination of $\gamma, \delta_{B}$ and $r_{B}$ in the charged modes and after full combination of the LHCb measurements.
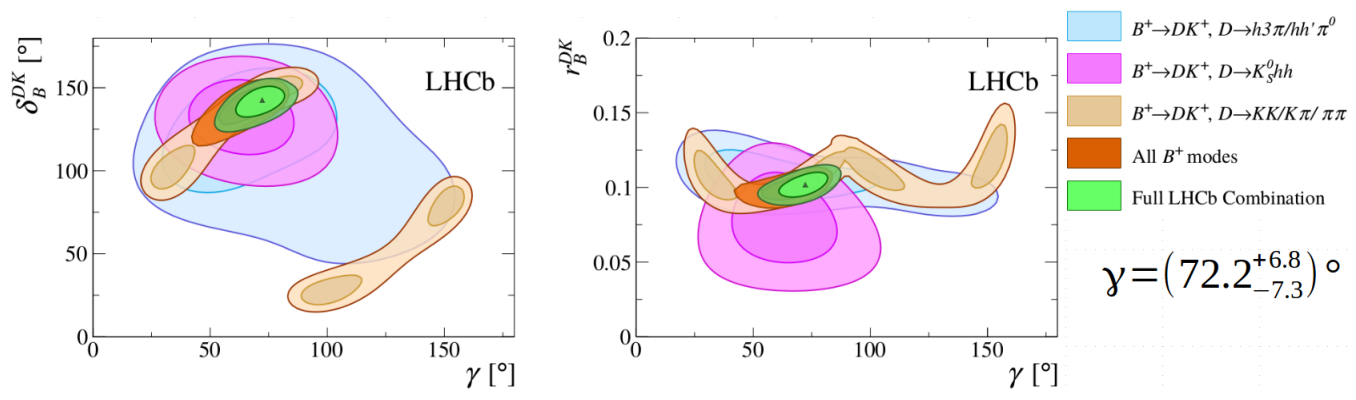

Figure 5: Determination of $\gamma$ altogether with $\delta_{B}$ and $r_{B}$ with the LHCb data in the different charged modes and after full combination of the LHCb measurements. 


\section{References}

[1] Joachim Brod, Jure Zupan, The ultimate theoretical error on gamma from $B \rightarrow D K$ decays, JHEP 01 (2014) 051

[2] Joachim Brod et al., New physics effects in tree-level decays and the precision in the determination of the quark mixing angle $\gamma$, Phys. Rev. D 92, 033002

[3] R. Aaij et al., the LHCb collaboration, Measurement of CP observables in $B^{ \pm} \rightarrow D K^{ \pm}$and $B^{ \pm} \rightarrow D \pi^{ \pm}$with two- and four-body D decays, Phys. Rev. B760 (2016) 117-131

[4] R. Aaij et al., the LHCb collaboration,A study of CP violation in $B^{\mp} \rightarrow D h^{\mp}(h=K, \pi)$ with the modes $D \rightarrow K^{\mp} \pi^{ \pm} \pi^{0}, D \rightarrow \pi^{+} \pi^{-} \pi^{0}$ and $D \rightarrow K^{+} K^{-} \pi^{0}$, Phys. Rev. D91 (2015) 112014

[5] M. Gronau and D. London, How to determine all the angles of the unitarity triangle from $D_{d}^{0} \rightarrow D K_{s}$ and $B_{s}^{0} \rightarrow D \phi$, Phys. Lett. B253 (1991) 483-488

[6] M. Gronau and D. Wyler, On determining a weak phase from charged B decay asymmetries, Phys. Lett. B265 (1991) 172-176

[7] David Atwood, Isard Dunietz, and Amarjit Soni, Enhanced CP Violation with $B \rightarrow K D^{0}\left(\bar{D}^{0}\right)$ modes and extraction of the Cabibbo-Kobayashi-Maskawa Angle $\gamma$, Phys. Rev. Lett. 78, 3257

[8] M. Nayak et al., First determination of the CP content of $D \rightarrow \pi^{+} \pi^{-} \pi^{0}$ and $D \rightarrow K^{+} K^{-} \pi^{0}$, Phys. Lett. B740 (2015) 1-7

[9] S. Malde et al., First determination of the CP content of $D \rightarrow \pi^{+} \pi^{-} \pi^{+} \pi^{-}$and updated determination of the CP contents of $D \rightarrow \pi^{+} \pi^{-} \pi^{0}$ and $D \rightarrow K^{+} K^{-} \pi^{0}$, Phys. Lett. B747 (2015) 9

[10] D. Atwood and A. Soni, Role of a charm factory in extracting CKM-phase information via $B \rightarrow D K$, Phys. Rev. D68 (2003) 033003

[11] T. Evans et al., Improved determination of the $D \rightarrow K^{-} \pi^{+} \pi^{+} \pi^{-}$coherence factor and associated hadronic parameters from a combination of $e^{+} e^{-} \rightarrow \psi(3770) \rightarrow c \bar{c}$ and $p p \rightarrow c \bar{c} X$ data, Phys. Lett. B757 (2016), 520-527

[12] R. Aaij et al., the LHCb collaboration, Measurement of the CKM angle $\gamma$ using $B^{ \pm} \rightarrow D K^{ \pm}$with $D \rightarrow K_{S} \pi^{+} \pi^{-}, K_{S} K^{+} K^{-}$decays, arXiv:1408.2748v2 [hep-ex]

[13] R. Aaij et al., the LHCb collaboration, A study of CP violation in $B^{ \pm} \rightarrow D K^{ \pm}$and $B^{ \pm} \rightarrow D \pi^{ \pm}$decays with $D \rightarrow K_{s}^{0} K^{ \pm} \pi^{ \pm}$final states, Phys. Lett. B733C (2014) 36-45

[14] M. Gronau, On determining a weak phase from charged B decay asymmetries, Phys. Lett. B265 (1991) 172-176

[15] R. Aaij et al., the LHCb collaboration, Measurement of the CKM angle $\gamma$ from a combination of LHCb results, JHEP 12 (2016) 087

[16] R. Aaij et al., the LHCb collaboration, Study of the decay $B^{ \pm} \rightarrow D K^{\star \pm}$ with two-body D decays, LHCb-CONF-2016-014 